\title{
Molecular mechanism of light responses in Neurospora: from light-induced transcription to photoadaptation
}

\author{
Qiyang He and Yi Liu' ${ }^{1}$ \\ Department of Physiology, The University of Texas Southwestern Medical Center, Dallas, Texas 75390, USA
}

\begin{abstract}
Blue light regulates many molecular and physiological activities in a large number of organisms. In Neurospora crassa, a eukaryotic model system for studying blue-light responses, the transcription factor and blue-light photoreceptor WHITE COLLAR-1 (WC-1) and its partner WC-2 are central to blue-light sensing. Neurospora's light responses are transient, that is, following an initial acute phase of induction, light-regulated processes are down-regulated under continuous illumination, a phenomenon called photoadaptation. The molecular mechanism(s) of photoadaptation are not well understood. Here we show that a common mechanism controls the light-induced transcription of immediate early genes (such as frq, al-3, and $v v d$ ) in Neurospora, in which light induces the binding of identical large WC-1/WC-2 complexes (L-WCC) to the light response elements (LREs) in their promoters. Using recombinant proteins, we show that the WC complexes are functional without the requirement of additional factors. In vivo, WCC has a long period photocycle, indicating that it cannot be efficiently used for repeated light activation. Contrary to previous expectations, we demonstrate that the light-induced hyperphosphorylation of WC proteins inhibits bindings of the L-WCC to the LREs. We show that, in vivo, due to its rapid hyperphosphorylation, L-WCC can only bind transiently to LREs, indicating that WCC hyperphosphorylation is a critical process for photoadaptation. Finally, phosphorylation was also shown to inhibit the LRE-binding activity of D-WCC (dark WC complex), suggesting that it plays an important role in the circadian negative feedback loop.
\end{abstract}

[Keywords: white collar-1; light; LOV domain; photoadaptation; phosphorylation; circadian]

Received August 29, 2005; revised version accepted September 26, 2005.

Light regulates a wide variety of physiological processes in fungi. Neurospora crassa is one of the best understood model organisms for understanding blue-light responses in fungi and blue-light sensing in general (Linden et al. 1997; Liu 2003; Liu et al. 2003). In Neurospora, all known light responses are mediated by blue light, and almost all responses require the protein products of two genes, white collar-1 (wc-1) and wc-2 (Degli Innocenti and Russo 1984; Lakin-Thomas et al. 1990; Linden et al. 1997; Collett et al. 2002; He et al. 2002; Lee et al. 2003). The cloning of the two wc genes by Macino and coworkers (Ballario et al. 1996; Linden and Macino 1997) revealed that they are both PER-ARNT-SIM (PAS) domaincontaining transcription factors with GATA type Zn-finger DNA-binding domains. WC-1 contains three PAS domains, while WC-2 has only one PAS domain. The most N-terminal PAS domain of WC-1 belongs to a specialized class of PAS domains known as LOV (light, oxygen, or voltage) domains (Crosthwaite et al. 1997; He

${ }^{1}$ Corresponding author.

E-MAIL Yi.Liu@UTSouthwestern.edu; FAX (214) 645-6049.

Articel published online ahead of print. Article and publication date are at http://www.genesdev.org/cgi/doi/10.1101/gad.1369605. et al. 2002; Cheng et al. 2003a). We and others have shown that, similar to the well-studied phototropins in plants (Briggs and Christie 2002), the LOV domain of WC-1 is associated with a flavin and functions as a bluelight photoreceptor in Neurospora (Froehlich et al. 2002; He et al. 2002). However, in contrast to phototropins, the LOV domain of WC-1 binds to FAD instead of FMN (flavin mononucleotide) and contains a significant extension in the loop connecting the $\alpha^{\prime} \mathrm{A}$ and $\alpha \mathrm{C}$ helices.

The WC proteins heterodimerize via their PAS domains to form a WC complex (WCC) in vivo (Ballario et al. 1998; Talora et al. 1999; Cheng et al. 2002, 2003a), and it is this complex that mediates light responses by activating transcription of light-inducible genes (Linden et al. 1997; Liu et al. 2003). Upon light exposure, the immediate light-inducible genes such as the circadian clock component frequency (frq), genes involved in carotenoid biosynthesis (albino, al-1-al-3; vivid, vvd), are induced to their peak levels in 15-30 min. Promoter analysis of the frq promoter revealed that the rapid light induction of frq transcription, a process that is critical for the light resetting of the circadian clock (Crosthwaite et al. 1995), is due to the light-induced binding of a large WCC (LWCC) to two imperfect GATN repeats, named light re- 
sponse elements (LREs) (Froehlich et al. 2002). The previously identified light response element (also called APE) of al-3 also contains two GATA repeats (Carattoli et al. 1994), but how light activates the transcription of al-3 and other immediate light-inducible genes is not known.

Phototropins, the plant blue-light photoreceptors mediating phototropism, are the best photochemically and structurally studied LOV-domain-containing photoreceptors (Briggs and Huala 1999; Christie et al. 1999; Crosson and Moffat 2001; Harper et al. 2003; Christie and Briggs 2005). Like many other photoreceptors, phototropins undergo rapid photocycles in the dark after their light activation to return to the dark state (Salomon et al. 2000), so that they can be repeatedly activated by light. Whether WC-1 has a photocycle and how its photochemical property affects its function in light responses are not known.

Photoadaptation (or so-called light adaptation) is a mechanism that organisms used to adjust the sensitivity of their photoreception systems to prolonged light exposure. In Neurospora, despite the continuous presence of light, levels of immediate light-induced genes quickly decrease to relatively low levels after $2 \mathrm{~h}$ in constant light (LL) (Schrott 1981; Arpaia et al. 1999; Cheng et al. 2001a; Schwerdtfeger and Linden 2001; Shrode et al. 2001). In addition, shifting Neurospora from low- to high-intensity light can trigger a second light induction (Arpaia et al. 1999; Schwerdtfeger and Linden 2001). These photoadaptative responses are typical for Neurospora and similar to adaptive responses in vertebrates (Pugh Jr. et al. 1999).

At the molecular level, the mechanism for photoadaptation is not well understood in Neurospora, although VVD and protein kinase $\mathrm{C}(\mathrm{PKC})$ have been implicated in this process (Arpaia et al. 1999; Heintzen et al. 2001; Schwerdtfeger and Linden 2001; Shrode et al. 2001; Franchi et al. 2005). VVD is a small protein consisting mostly of a single LOV domain and is another blue-light photoreceptor (Cheng et al. 2003a; Schwerdtfeger and Linden 2003) that acts as a repressor of light responses. PKC affects light response probably by negatively regulating the levels of WC-1 protein (Franchi et al. 2005).

WCs autoregulate their own transcription and their post-translational modifications (Ballario et al. 1996; Talora et al. 1999; Schwerdtfeger and Linden 2000). wc-1 is an immediate early light-inducible gene. Both WCs are phosphorylated proteins in the dark and become hyperphosphorylated after a light pulse. The light-induced WC hyperphosphorylation was previously proposed to have two roles. First, it may trigger WC-1 degradation, as WC-1 is less stable in the light than in the dark (Talora et al. 1999; Lee et al. 2000; Schwerdtfeger and Linden 2000; Franchi et al. 2005). Second, it was proposed to be a mechanism that activates WCC, since WC-1 hyperphosphorylation appears to correlate with the activation of transcription. Consistent with this, WC-1 stayed hyperphosphorylated in LL in $v v d$ mutants (Heintzen et al. 2001; Schwerdtfeger and Linden 2001). However, there is no direct evidence supporting the role of light-induced hyperphosphorylation in activating WCC activity. On the contrary, the correlation between the extent of WC-1 hyperphosphorylation and the levels of the light-induced mRNAs can also be viewed as such that the WC-1 hyperphosphorylation coincides with the decrease of the mRNA levels. In this view, the light-induced hyperphosphorylation of the WCs could be inhibitory to its activity.

In addition to their essential roles in light responses, both WC proteins are also the positive elements of the circadian feedback loop in Neurospora, essential for clock function. In the dark, a heterodimeric WCC (DWCC) binds to the C-box (the distal LRE) of the frq promoter, activating its transcription (Crosthwaite et al. 1997; Cheng et al. 2001b; Froehlich et al. 2003; Dunlap and Loros 2004). On the other hand, FRQ and FRH (FRQinteraction RNA helicase), the negative elements in the loop, repress the transcription of frq by inhibiting WCC activity (Aronson et al. 1994; Cheng et al. 2001a, 2005; Denault et al. 2001). The WC phosphorylation in the dark appears to be an important event in this process. Previously, we have shown that the light-independent WC-1 phosphorylation negatively regulates its function in the circadian feedback loop (He et al. 2005), a notion that is further supported by a very recent study (Schafmeier et al. 2005).

Here we set out to further elucidate the molecular mechanism of light responses in Neurospora and the regulation of WCC. We show that the light induction of immediate light-inducible genes is regulated by a common mechanism through binding to their promoter LREs by identical L-WCCs. Using recombinant WCC expressed from insect cells, we show that the WCCs consist of only WC-1 and WC-2 proteins. Unlike the phototropins, WCC has a long period photocycle in vivo, consistent with Neurospora's known refractory time in response to a second light pulse. Furthermore, we demonstrate that the light-dependent and light-independent phosphorylation of WCC inhibit bindings of both L-WCC and D-WCC to the LREs. Thus, phosphorylation of WCC should be an important part of the photoadaptation mechanism and a critical process in the circadian negative feedback loop.

\section{Results}

Light-induced binding of the identical L-WCC to the LREs of the frq, vvd, and al-3 genes

Two LREs (proximal and distal), each containing two imperfect GATN repeats, were previously identified in the frq promoter to mediate its light-inducible transcription (Froehlich et al. 2002). To determine how other immediate light-inducible genes are regulated in Neurospora, we examined the binding of WCC to the promoter elements of the $v v d$ (Heintzen et al. 2001) and al-3 genes. Like $f r q, v v d$ and al-3 mRNA levels are induced to their peak levels $\sim 15$ min after light exposure (Baima et al. 1991; Carattoli et al. 1994; Crosthwaite et al. 1995; Heintzen et al. 2001). However, these three genes have different requirements of WC-1 levels for their light-in- 
duced transcription (Cheng et al. 2003a,b; Lee et al. 2003; Liu 2003; Liu et al. 2003). While light induction of al-3 is very sensitive to changes of WC-1 levels, near normal light induction of $f r q$ can be achieved with very low levels of WC-1. The requirement of WC-1 for light induction of $v v d$ is between those two. Thus, these three genes should represent the full range of light sensitivity of immediate light-inducible genes in Neurospora.

An al-3 LRE element, containing two GATA repeats, was previously identified to be required for its light induction by deletion analysis of the al-3 promoter (Carattoli et al. 1994). By analyzing the promoter sequence of the $v v d$ promoter, we identified an LRE-like element located $\sim 400$ base pairs (bp) upstream from the translational start codon, containing two GATC repeats (Fig. 1A). Comparison of the four LREs from $f r q, a l-3$, and $v v d$ shows that the spacing between the two GATN motifs in each LRE varies significantly. While the fourth positions of the GATN motifs are different among different LREs, the same nucleotide is used for both GATN repeats in each. In addition, the fifth position of the first GATN motif and the nucleotide that precedes the second GATN motif are invariably a cytosine. Thus, a consensus sequence of the LRE appears to be GATNC----CGATN, in which $\mathrm{N}$ can be any nucleotide but the same nucleotide is used in both repeats.

We then examined the ability of WCC to bind these LREs using electrophoretic mobility shift assays (EMSAs). Using a $w c-2^{k o}$, Myc-His-WC-2 strain, we obtained partially purified WCC by nickel-column purification from cultures grown in constant darkness (DD). The purified protein extracts were either kept in DD or exposed to a 10-min light pulse (LP) in vitro before they were incubated with different LRE probes. As shown in Figure 1B using the frq LRE (distal), the DD sample mostly resulted in a fast mobility gel shift (dark WCC, D-WCC), and the LP treatment led to the formation of a slower mobility protein-LRE complex. Such gel-shifts were supershifted by the WC-1, WC-2, or the monoclonal c-Myc antibodies, and were competed away by cold frq probe but not by a cold probe with a mutated GATN motif. These results are very similar to those previously reported (Froehlich et al. 2002) and indicate that light induces a large WCC (Light WCC, L-WCC) to bind to the frq LRE. The bands lower than the D-WCC are likely due to unspecific banding of the probe by the antiserum.

Similar EMSAs were performed using the $v v d$ and al-3 LRE probes. As shown in Figure 1C and D, LP also induced the binding of an L-WCC to these two LREs, and such bindings can be supershifted with the WC antibodies (data not shown) and can be specifically competed away by the cold LRE probes. These data suggest that these two promoter elements are, indeed, the LREs that mediate the light-induced transcription of these two genes. The D-WCC was also found to bind to the $v v d$ and al-3 LREs in the dark, perhaps explaining the fact that these genes are also clock-controlled genes (Arpaia et al. 1995; Heintzen et al. 2001).

The gel mobilities of the L-WCC/LRE complexes are similar for these three different LRE probes, suggesting
A

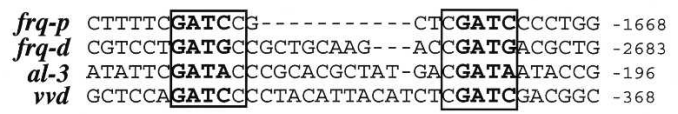

B

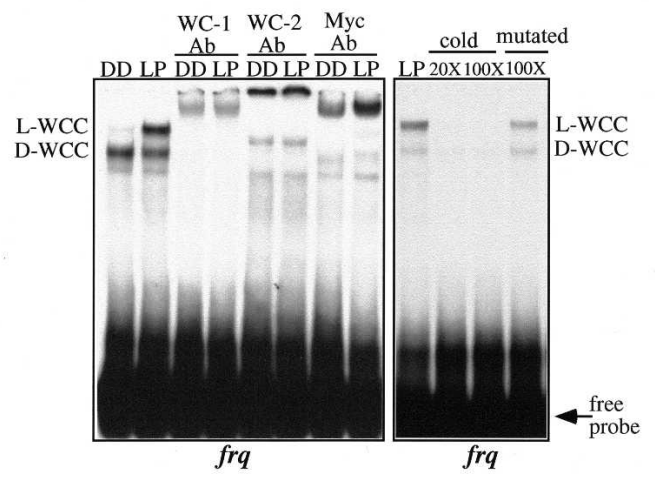

$\mathrm{C}$

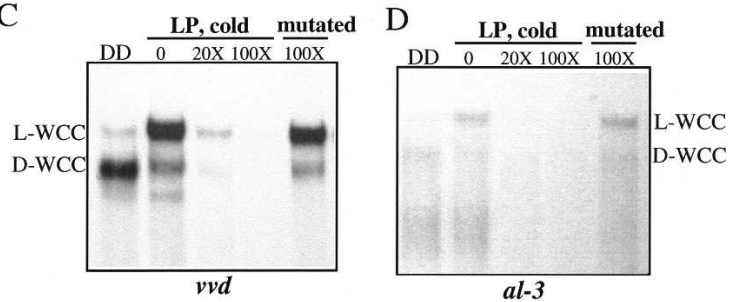

E

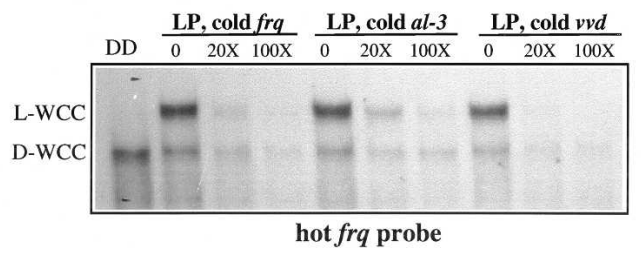

Figure 1. Light induces the binding of identical L-WCCs to the LREs of frq, $v V d$, and al-3. (A) DNA sequence alignment of LREs in the promoters of $f r q, a l-3$, and $v v d$. (frq-p) frq proximal LRE; (frq-d) frq distal LRE. (B-D) EMSA assays showing the binding of the WCC to the LREs of $f r q$ (distal) $(B), \operatorname{vvd}(C)$, and $a l-3(D)$. In some of EMSA assays, cold wild-type or mutated probes were added, and in some, antibodies against the WC proteins were used. (E) EMSA assay showing that the binding of the L-WCC to the frq LRE (radioactive labeled) can be efficiently competed away by the cold LREs of al-3 and $v v d$. For all EMSA experiments described in this figure, partially purified WCC from Neurospora (DD culture) was used. The LPs (10 $\mathrm{min}$ ) were administered in vitro.

that they are likely the same L-WCC complex. To examine this, we performed competitive EMSA assays. As shown in Figure 1E, the formation of the L-WCC/LRE (frq) complex can be efficiently competed away by the cold al-3 or vvd probe, indicating that identical L-WCC complexes bind to different LREs after light exposure. Thus, most, if not all, of the immediate light-inducible transcription in Neurospora is regulated in the same manner.

Recombinant WCC expressed in insect cells is sufficient for mediating the light-dependent binding to the LRES

The L-WCC is larger than the D-WCC. Although WC-1 is known to self-associate (Cheng et al. 2003b), it is pos- 

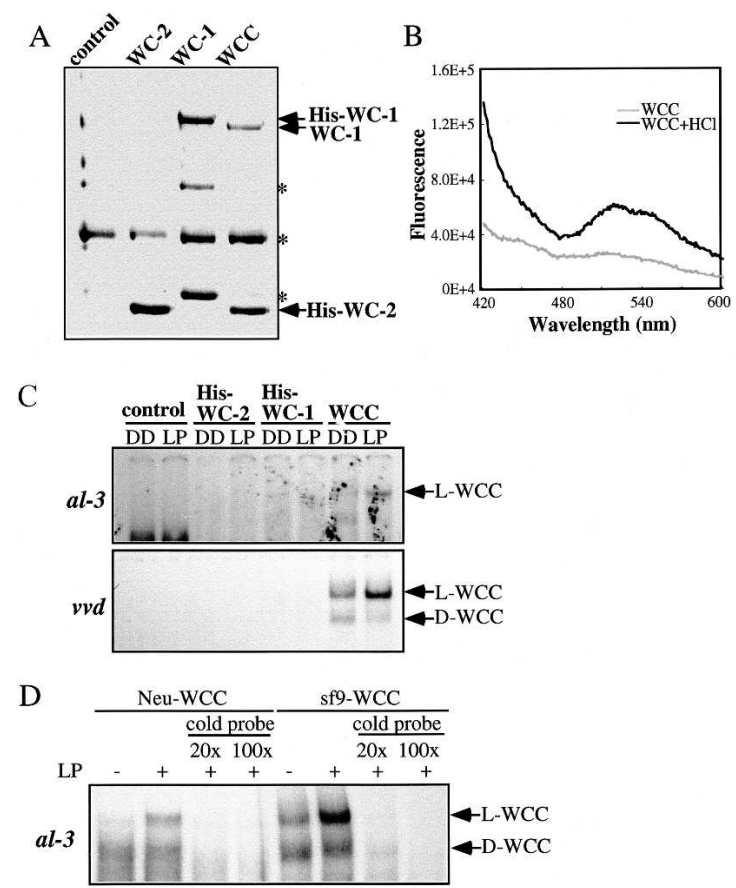

Figure 2. The recombinant sf9 insect-cell-expressed WCC is sufficient to mediate the light-induced binding to the LREs. $(A)$ SDS-PAGE gel showing the nickel column purification products of His-WC-1, His-WC-2, and the complex of WC-1 and HisWC-2 from insect cells (grown in DD) infected by respective viruses. (Control) Mock purification from uninfected cells. The asterisks indicate a nonspecific band and the possible His-WC-1 degradation products. (B) Fluorescence emission spectra analysis showing the presence of FAD in the purified WCC from insect cells. $(C)$ EMSA assay showing the induction of the LWCC/LRE (al-3 or $v v d$ ) complexes after an LP in vitro. (D) EMSA assay showing the side-by-side comparison of the WCC/ LRE complexes (al-3 probe) using WCC purified from Neurospora or insect cells.

sible that novel proteins may be present in the L-WCC to mediate the light response. To examine whether the WC proteins themselves are sufficient to mediate light-induced transcription and to examine whether there are other components in the L-WCC, we expressed and purified the full-length recombinant WC-1, WC-2, or WCC in the dark using an insect cell baculovirus expression system (Fig. 2A). The WCC was expressed by cotransfecting both WC-1 and His-WC-2 viruses and purified using a nickel column. Fluorescence spectroscopic and thin layer chromatography analyses showed that the purified WCC contained FAD (Fig. 2B; data not shown). However, the comparison of the FAD levels to that of the WCC showed that only $5 \%-10 \%$ of the insect-cell-expressed WCC contained the chromophore, suggesting that only small amounts of the complex are functional.

EMSAs using these purified proteins were carried out in DD or after an LP in vitro. As shown in Figure 2C, only the WCC was able to bind LREs and induce the L-WCC/LRE complexes. Side-by-side EMSA comparison using the purified WCC from Neurospora and sf9 cells showed that the gel mobilities of the WCC/LRE com- plexes were identical. These data indicate that the WCC alone is sufficient to mediate its light-induced and lightindependent LRE binding and there is likely no other component in the WCCs in vivo. This is consistent with the previous results using in vitro translated WC proteins (Froehlich et al. 2002).

\section{WCC purified from Neurospora does not have an active photocycle in vitro}

After activation by light, phototropins undergo rapid photocycles (from seconds to minutes) and return to the dark state, so that they can be repeatedly activated by light (Christie et al. 1999; Salomon et al. 2000; Briggs and Christie 2002; Harper et al. 2003). Since only a small portion of the WCC purified from the insect cells contained FAD, this prevented us from carrying out further photochemical analysis of the complex in vitro. To examine whether the WCC has a photocycle, the Neurospora purified WCC was used in EMSA to check whether it can revert back to the dark state in DD after light exposure. After a 10-min light pulse, the purified WCC samples were incubated in DD for various periods before they were mixed with the frq LRE probe. If there is an active photocycle, the L-WCC should be converted back to the dark state and can no longer bind to the DNA. As shown in Figure 3, the duration of dark incubation up to $4 \mathrm{~h}$ did not significantly reduce the level of the L-WCC/ LRE complex. Similar results were also obtained using the vvd and al-3 LRE probes (data not shown). These results indicate that in vitro, most of the Neurospora WCC, once activated by light, will stay activated and cannot be converted back to the dark state. Thus, WC-1 does not appear to have an active photocycle as do the phototropins. The lack of an active WC-1 photocycle means that it cannot be repeatedly activated by light, which is consistent with previous results showing that Neurospora is insensitive to a second light pulse given
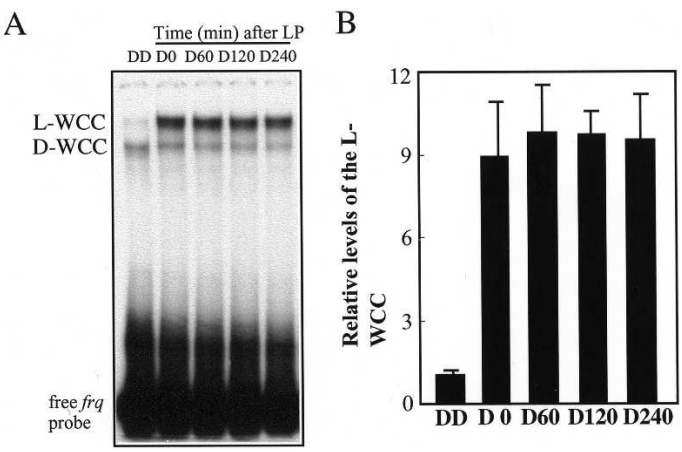

Figure 3. Most of the L-WCC cannot recover back to the dark state after light activation in vitro. (A) EMSA assay showing the binding of the WCC to the frq LRE after a 10-min in vitro LP. Partially purified WCC from Neurospora (DD culture) was used. After the LP, some of the samples were incubated in the dark for the indicated time (in minutes) before they were mixed with the LRE probe. $(B)$ Densitometric analysis of the result in $A$ and several independent experiments. 
$<1 \mathrm{~h}$ after the first one (Schrott 1981; Arpaia et al. 1999; Schwerdtfeger and Linden 2001).

\section{In vivo, WCC undergoes a photocycle with a long period}

We then asked whether WC-1 undergoes a photocycle in vivo. If there is a photocycle, the same WC-1 that has been activated by the first LP should be able to trigger light responses by a second LP. To test this, we examined the light-induced WC-1 hyperphosphorylation and lightinduced transcription of a wild-type strain by a second LP (15 min) administered $4 \mathrm{~h}$ after the first one (Fig. 4A). To block the production of the newly synthesized WC-1 protein, the protein synthesis inhibitor cycloheximide (CHX, $50 \mu \mathrm{M})$ was added to the cultures. As shown in Figure $4 \mathrm{~B}$ in cultures with/without $\mathrm{CHX}$, the first LP induced hyperphosphorylation of WC-1 and transcription of the immediate light-inducible genes (lanes 2,3), including $v v d, a l-3$, and $w c-1$. A high light intensity was used for the first LP ( 3500 lux), so that all WC-1 proteins should be activated. CHX treatment appeared to have a general and nonspecific effect on the levels of mRNAs, perhaps due to its role in blocking translation and stabilizing mRNA. Four hours after the first LP (Fig. $4 \mathrm{~B}$, lane 5), WC-1 resumed its normal phosphorylation state in DD in both sets of samples. For the CHX-treated samples, the first LP also led to $40 \%-50 \%$ reduction of the WC-1 level (Fig. 4B, cf. lanes 1 and 5, our WC-1 antibody is more sensitive to the hyperphosphorylated WC-1 species than the hypophosphorylated forms), indicating that light triggered the degradation of a portion of WC-1, consistent with previous results (Talora et al. 1999; Lee et al. 2000; Franchi et al. 2005). In addition, there was an increase of the least phosphorylated WC-1 species after $4 \mathrm{~h}$ in DD for the CHX-treated samples (Fig. 4B, cf. lanes 4 and 5), indicating an action of WC-1 dephosphorylation. Fifteen minutes after the second LP, small but consistent inductions of WC-1 hyperphosphorylation and gene transcription of $v v d, a l-3$, and $w c-1$ were observed (Fig. 4B, lane 6, CHX samples). Since these light responses require $\mathrm{WC}-1$ and there was no newly synthesize WC-1, these data suggest that WC-1 is capable of being reactivated by light. Thus, it has a photocycle in vivo, albeit with a long half-life.

To further confirm this conclusion and to exclude the potential side-effects of $\mathrm{CHX}$, we used a $w c-1^{R I P}$, qaWC-1 strain, in which $w c-1$ is under the control of the quinic acid (QA) inducible promoter. In the absence of QA, there is no detectable WC-1 by Western blot analysis (Cheng et al. 2001b). To examine the ability of WC-1 to be repeatedly activated by light pulses, the cultures were first grown in DD in the presence of QA to induce WC-1 expression, and then QA was removed $2 \mathrm{~h}$ before the first LP. After the first LP (10 min), the cultures were maintained in DD for 1-3 h before a second LP (Fig. 4C).

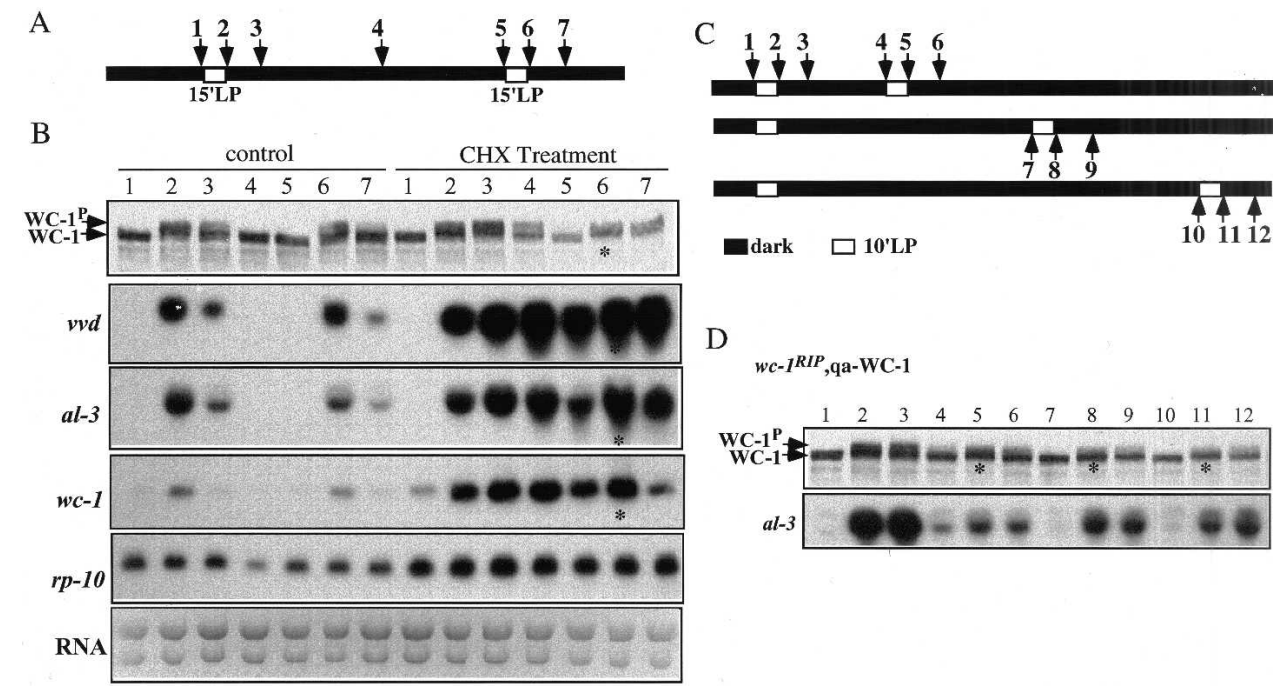

Figure 4. Two pulse experiments demonstrate that WC-1 has a long-period photocycle in vivo. (A) A schematic diagram depicts the time points used to harvest Neurospora cultures for the experiment shown in B. A wild-type Neurospora strain was used. (1) DD. (2) Immediately after the first LP. (3) Thirty minutes after the first LP. (4) Two hours after the first LP. (5) Four hours after the first LP. (6) Immediately after the second 15-min LP. (7) Thirty minutes after the second LP. (B) Western and Northern blot analyses showing that the second LP can result in light-induced WC-1 hyperphosphorylation and light-induced transcription. CHX was added to half of the culture to block protein synthesis. $(r p-10)$ A ribosomal protein gene that was used as a negative control for light responses in the Northern blot analysis. $(C)$ A schematic diagram depicts the time points used to harvest Neurospora cultures for the experiment shown in $D$. The second LP was administered 1,2, or $3 \mathrm{~h}$ after the first LP. (1) DD. $(4,7,10)$ One, two, and three hours in DD after the first LP, respectively. $(2,5,8,11)$ Immediately after the first and the second LP. $(3,6,9,12)$ Thirty minutes after the first or the second LP. $(D)$ Western and Northern blot analyses showing that the second LP can result in light-induced WC-1 hyperphosphorylation and lightinduced al-3 transcription. The $w c-1^{R I P}$, qa-WC-1strain was used. The culture was first grown in medium containing QA for $1 \mathrm{~d}$ before it was removed by washing the cultures with fresh minimal medium containing $2 \%$ glucose. The first LP was administered $2 \mathrm{~h}$ after the removal of QA. 
As shown in Figure 4D, the second LP resulted in WC-1 hyperphosphorylation and induction of al-3 transcription (Fig. 4D, lanes 5,8,11). The second LP administered $2 \mathrm{~h}$ after the first LP resulted in more light induction of al-3 than that after only $1 \mathrm{~h}$, suggesting that the WC-1 photocycle is $>1 \mathrm{~h}$. Together, these data demonstrate that despite its long half-life, WC-1 does have a photocycle in vivo. Such a slow photocycle means that WC-1 cannot be efficiently used for multiple photoactivations. Thus, despite the presence of significant amounts of WC-1 in LL, most cannot be activated by light to mediate light-induced transcription.

WCC transiently binds to the promoters of the immediate light-inducible genes

When Neurospora is transferred from DD to LL, the immediate light-inducible genes will be rapidly induced, reaching their peak levels $\sim 15 \mathrm{~min}$ in LL. Afterward, their mRNA levels quickly decrease and stay at low levels (the levels are higher than the basal levels in DD) in LL, a process called photoadaptation. As shown above, the WCC has a slow photocycle in vivo, and in vitro, after its activation by light, it stays activated. Thus, if there is no other process(es) regulating its DNA-binding activity in vivo, we would expect that the L-WCC should bind to the LREs constantly in LL. To test this possibility, we performed chromatin immunoprecipitation (ChIP) assays to examine the binding of the WCC to the LREs ( $v v d, a l-3$, and $\left.f_{r q}\right)$ in vivo after a dark-to-light transition. Our WC-2 antibody was used for the immunoprecipitation. As shown in Figure 5, the light activation of WCC association with the LREs was transient, reaching their peaks after $15 \mathrm{~min}$ in LL. Afterward, the levels of the binding quickly decreased to low levels in LL. Significant WCC binding was also found for the frq LRE in the dark, consistent with frq being directly regulated by D-WCC in DD (Crosthwaite et al. 1997; Cheng et al.

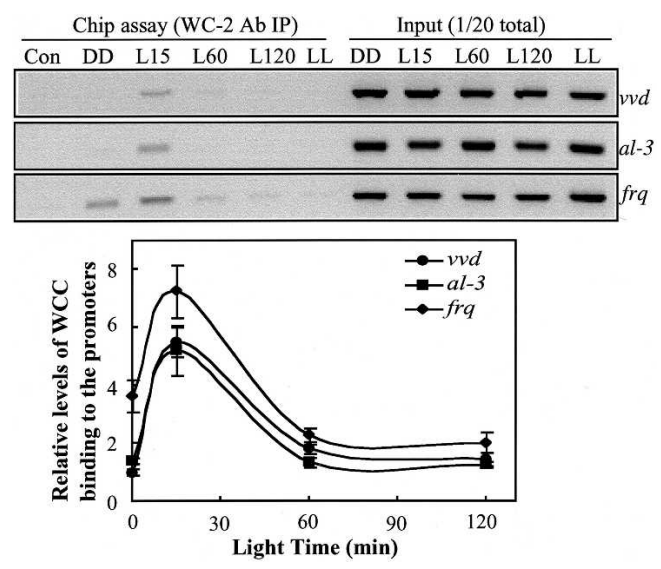

Figure 5. The L-WCC binds to the LREs transiently after transfer from DD to LL. ChIP assay using WC-2 antibody showing the binding of WCC to the LREs of $v v d$, al-3, and frq. The densitometric analysis of three independent experiments is shown in the bottom panel. 2001b; Froehlich et al. 2003). These data indicate that the light-induced binding of WCC to the LREs correlates with the light induction of transcription, and that there is an unknown mechanism negatively regulating the activity of WCC after light exposure.

\section{Light-induced WC-1 hyperphosphorylation does not correlate with promoter binding}

The dark-to-light transition results in transient hyperphosphorylation of WC-1 and WC-2, peaking after 15-30 min in LL (Talora et al. 1999; Schwerdtfeger and Linden 2000; Cheng et al. 2003a; He et al. 2005). In addition, WC-1 becomes less phosphorylated after prolonged exposure in LL, but its phosphorylation level is more extensive than that in DD (Schwerdtfeger and Linden 2001; Cheng et al. 2003a). Although the degradation of WC-1 by light could partially contribute to the photoadaptation process, the effect of light on WC-1 level is only modest and cannot explain the dramatic decrease of the mRNA levels after $30 \mathrm{~min}$ in the light. Previously, WC hyperphosphorylation was proposed to be an activation mechanism for WCC activity.

To understand the function of the light-induced WC hyperphosphorylation, we performed a detailed time course to examine the relationship between the lightinduced WC-1 hyperphosphorylation and the light-induced mRNA levels. As shown in Figure 6A, both WC-1 hyperphosphorylation and light-induced mRNA levels reached their peaks after $15 \mathrm{~min}$ in LL, and their levels quickly decreased afterward. Although light induction of these genes all showed clear photoadaptation, the kinetic profile of $v v d$ is different from those of al-3 and $w c-1$. The light activation of $v v d$ is prolonged in $\mathrm{LL}$, while the levels of al-1 and wc-1 drop to near very low levels in $90 \mathrm{~min}$. The kinetic of frq light induction is similar to that of the vvd (Collett et al. 2002; Schwerdtfeger and Linden 2003).

Clear increases of mRNA levels were seen after $5 \mathrm{~min}$ in LL, a time when WC-1 hyperphosphorylation was not obvious. At $30 \mathrm{~min}$ in LL, while WC-1 was still extensively hyperphosphorylated, the mRNA levels were already in the process of rapid decline. When taken into account the time needed for gene transcription and kinetics of the mRNA levels, the peak of WCC activity must occur before $15 \mathrm{~min}$ in LL. Thus, the peak of WC-1 hyperphosphorylation does not appear to correlate with the peak of WCC activity.

We then performed ChIP assays to determine the peak time of WCC binding to the LREs in vivo. As expected, the binding of WCC to the LREs was seen 2 min after light exposure, reached its peak at $5 \mathrm{~min}$, and decreased afterward (Fig. 6B). The rapid binding of L-WCC to the LRE was further confirmed by EMSA using nuclear extracts. As shown Figure 6C, the nuclear extracts prepared from the culture after 2 min of light exposure (lacking hyperphosphorylation) resulted in more L-WCC/LRE complex than the extracts from later time points. The time difference between the ChIP and EMSA assays is likely due to the time needed for the activated WCC to 
A

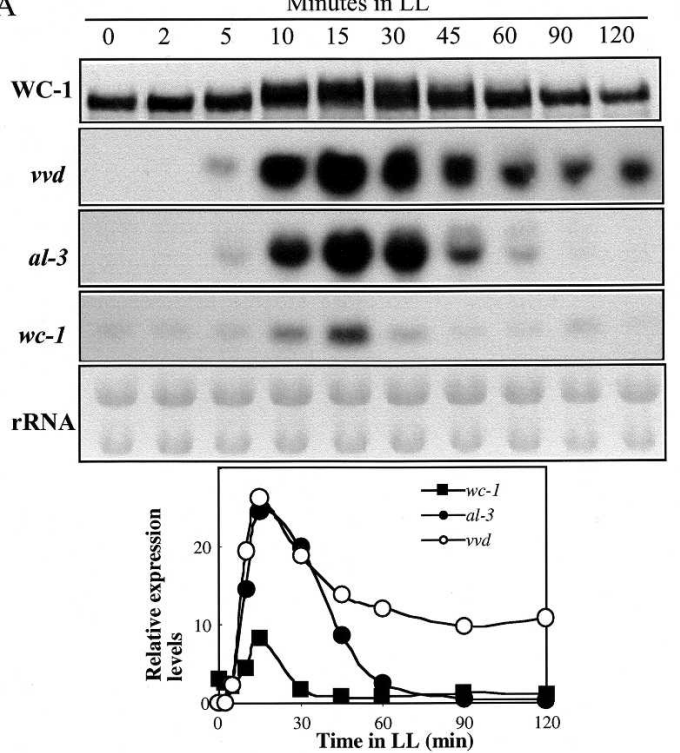

B

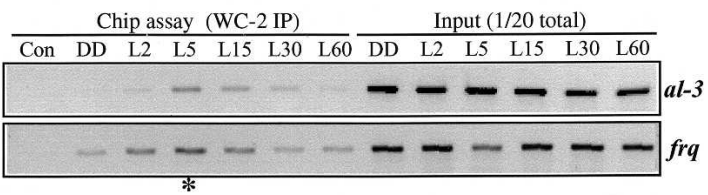

$\mathrm{C}$

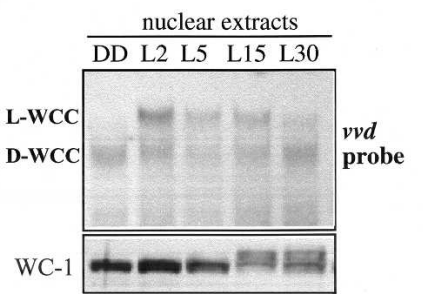

Figure 6. After the transfer from DD to LL, the peaks of LWCC binding to the LREs are not correlated with the peak of WC-1 hyperphosphorylation. (A) Western and Northern blot analysis showing the kinetics of the light-induced WC-1 hyperphosphorylation and the light induction of $v v d, a l-3$, and $w c-1$ mRNA after the dark-to-light transfer. Densitometric analysis of the Northern blot experiment is shown in the bottom panel. $(B)$ ChIP assay showing the binding kinetics between WCC and LREs $(a l-3$ and $f r q)$. (C) EMSA assay showing the binding kinetics between L-WCC and the vVd LRE. Nuclear extracts purified from cultures harvested in the indicated time points (minutes) were used in the assay. The bottom panel is the Western blot result showing the $\mathrm{WC}-1$ protein for these samples.

find its targets in vivo. Together, these data indicate that WCC binds to its targets rapidly after its activation by light and the hyperphosphorylation state of WC-1 does not correlate with the DNA-binding ability of WCC. Thus, it is unlikely that the light-induced WC-1 hyperphosphorylation has a stimulatory role on WCC activity.

\section{Phosphorylation of WCC inhibits the binding of both L-WCC and D-WCC to the LRES}

To demonstrate the role of the WC phosphorylation in regulating its activity, WCC was partially purified from a Neurospora culture that had been exposed to light for 15 min. Afterward, part of the purification products was treated with $\lambda$-phosphatase before the EMSA assay. As shown in Figure 7A, the dephosphorylation of WCC led to significant increases of both L-WCC and D-WCC/LRE $(v v d)$ complexes, effects that could be completely blocked by the addition of phosphatase inhibitors. A similar finding was also observed for the frq LRE probe (Fig. 7B). These results indicate that phosphorylation of WCC inhibits the DNA-binding activity of both L-WCC and D-WCC.

Inhibition of the activity of D-WCC by phosphorylation is consistent with our recent results showing that the mutation of the WC-1 light-independent phosphorylation sites positively regulates WCC activity in the dark (He et al. 2005). It also provides direct biochemical support for an important role for the WCC dark phosphorylation in the circadian negative feedback loop (He et al. 2005; Schafmeier et al. 2005).

To further demonstrate the role of the light-induced WCC hyperphosphorylation in inhibiting L-WCC binding to the LREs, we performed EMSA using WCC puri-
A

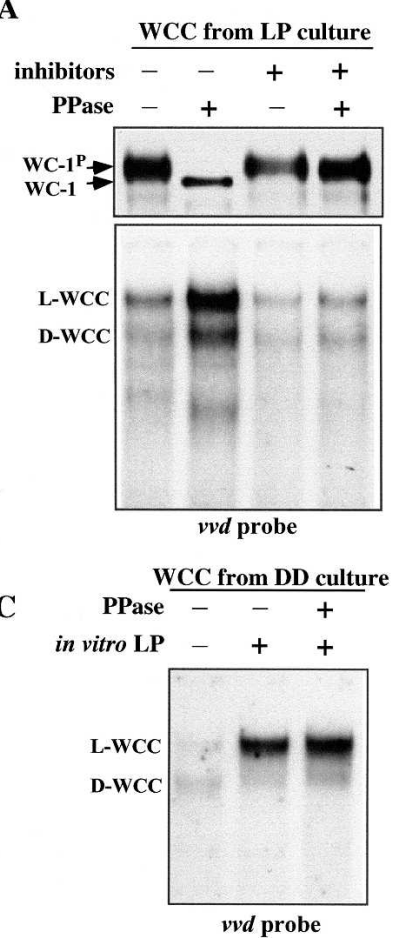

B
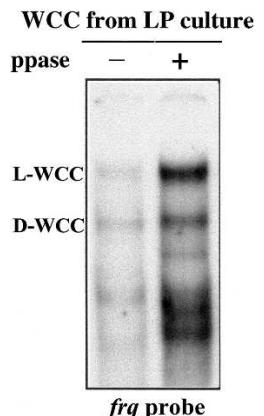

Figure 7. Dephosphorylation of WCC significantly enhances the binding of both L-WCC and D-WCC to the LREs. $(A, B)$ EMSA assays showing the binding of purified Neurospora WCC to the LREs $(v v d$ or $f r q)$ with/without the $\lambda$-phosphatase treatment. The cultures were harvested after a 15-min LP. All samples contained phosphatase buffer and were treated by the same procedure except the presence of phosphatase or phosphatase inhibitors. The top panel of $A$ is the Western blot result showing the phosphorylation profile of WC-1 used in the EMSA assay. (C) EMSA assay showing the binding of L-WCC to the vvd LRE after an LP in vitro. The WCC was purified from cultures grown in DD. 
fied from a culture grown in DD, which lacks the lightinduced hyperphosphorylation. If the inhibition of L-WCC DNA binding by phosphorylation shown above is mostly due to the light-induced WC hyperphosphorylation, for an LP given in vitro, we expect that the PPase treatment of WCC from DD should not significantly affect the DNA binding of the L-WCC. As shown in Figure $7 \mathrm{C}$, the PPase treatment only resulted in a small increase of the formation L-WCC/LRE complex, in contrast with the strong effects shown in Figure 7A and B. Thus, the light-induced hyperphosphorylation of WCC is responsible for most of the inhibition of L-WCC binding we observed in Figure 7A and B. Together, these data indicate that the light-induced WC phosphorylation negatively regulates the activity of L-WCC, which should result in its transient binding to the LREs and the decrease of transcription of light-inducible genes $15 \mathrm{~min}$ after the light exposure. Therefore, this post-translational regulation should be an important process mediating photoadaptation in Neurospora.

\section{Discussion}

A common mechanism regulating the light induction of immediate early genes

The WC proteins mediate almost all known light responses in Neurospora, and WC-1 is the blue-light photoreceptor. In this study, we showed that al-3 and $v v d$, two immediate-early light-inducible genes, are regulated in the same manner as previously demonstrated for frqlight-induced identical L-WCCs to bind to the LREs in their promoters (Froehlich et al. 2002). Thus, despite their different requirements of WC-1 levels for their light inductions (Liu et al. 2003), this mechanism should apply to all immediate early light-inducible genes in this organism. The different requirements of WC-1 levels of different genes may be due to different promoter contexts of the LREs (which may affect the affinity between L-WCC and LRE) or the number of LREs in their promoters. Among known immediate-early genes, frq is least sensitive to changes in WC-1 levels, probably because it has two LREs in its promoter (Froehlich et al. 2002), allowing it to more efficiently recruit the L-WCC in vivo.

Using recombinant WCC from insect cells, we showed that both the D-WCC and the L-WCC consist of only WC-1 and WC-2 proteins. This is consistent with the earlier result using in vitro translated WC proteins made from the rabbit reticulocyte lysate system (Froehlich et al. 2002, 2003). Therefore, the L-WCC should be the result of multimerization of the WC proteins, a notion that is supported by the observation of WC-1 self-association in vivo (Cheng et al. 2003b). However, it is possible that other factor(s) may interact with the WCCs to regulate their activities before their binding to the LREs.

\section{Long period photocycle of WC-1}

In vitro, the persistent activation of L-WCC in DD after an LP (Fig. 3) suggests that, unlike the phototropins, most of the WC-1 cannot recover back to the dark state after its photoactivation. The in vivo experiments showed that, although Neurospora is insensitive to a second light if given $<1 \mathrm{~h}$ of dark recovery time (Schrott 1981; Arpaia et al. 1999; Schwerdtfeger and Linden 2001), WCC can respond to a second light pulse after $>1$ $\mathrm{h}$ in DD (Fig. 4). These data, although indirect, suggest that WC-1 has a long period photocycle $(>1 \mathrm{~h})$. This is likely due to the formation of long-lived photoactivated intermediate in its LOV domain, which involves the formation of a covalent bond between the critical and highly conserved cysteine residue of the LOV domain and the flavin chromophore (Salomon et al. 2000; Crosson and Moffat 2002; Crosson et al. 2003; Harper et al. 2003). Such a photochemical characteristic of WC-1 will render most of the proteins incapable of being reactivated by light and will have a major impact on its function (see below).

The Arabidopsis FKF1 (Flavin-binding, Kelch repeat, F-box) protein and its homologs are another class of LOV-domain-containing blue-light receptors in plants mediating circadian and photoperiodic responses (Somers et al. 2000; Cheng et al. 2003a; Imaizumi et al. 2003; Mas et al. 2003). Studies of the recombinant FKF1 protein showed that it does not have appreciable dark recovery in vitro. Thus, these LOV-domain-containing proteins, like WC-1, also do not have active photocycles. Although FKF-1 and its related homologs bind to FMN, they and WC-1 share a significant extension in the loop connecting the $\alpha^{\prime} \mathrm{A}$ and $\alpha \mathrm{C}$ helices, a feature probably contributing to their common photochemical behavior.

\section{Photoadaptation in Neurospora}

Photoadaptation mechanisms allow organisms to adjust their physiological activities to prolonged light exposure. In Neurospora, after the initial rapid induction of lightinducible genes, the responses quickly decrease and stay at low levels (Schrott 1981; Arpaia et al. 1999; Heintzen et al. 2001; Schwerdtfeger and Linden 2001; Shrode et al. 2001), the results of light insensitivity. Similar to the kinetics of the induction of immediate-early genes, WC proteins first become hyperphosphorylated after 15-30 min in LL, and then the levels of their phosphorylation decrease and are maintained at levels that are higher than those in DD (Talora et al. 1999; Schwerdtfeger and Linden 2000, 2001; Cheng et al. 2003a; He et al. 2005).

Based on previous studies and the results of this study, photoadaptation in Neurospora is mediated by several regulations. First, light triggers the degradation of part of the WC-1 pool, a process probably mediated by its hyperphosphorylation (Fig. 4; Talora et al. 1999; Lee et al. 2000). We estimate that $\sim 40 \%-50 \%$ of WC- 1 is degraded through this process. Phosphorylation-dependent protein degradation has been demonstrated for FRQ in Neurospora, a critical process regulating the circadian clock (Liu et al. 2000; Gorl et al. 2001; Yang et al. 2002, 2004; He et al. 2003).

Second, as we demonstrated in this study, contrary to previous expectations, the light-induced WC hyperphos- 
phorylation inhibits the DNA-binding ability of the LWCC (Fig. 7). This inhibition should lead to transient binding of the L-WCC to the LREs and the decrease of the transcription of the light-inducible gene after 15-30 min in LL (Fig. 6). Afterward, the light activation of the WCC and the light-induced WCC phosphorylation probably reach a balance in LL so that the transcription of light-inducible genes is maintained at levels that are higher than those in DD but are significantly lower than the initial light responses. The sites of the light-induced WC phosphorylation and the kinase(s) involved are not known. PKC was previously implicated as a kinase that phosphorylates WC-1 and down-regulates its levels, but it was thought not to be the kinase that mediates the WC-1 hyperphosphorylation (Arpaia et al. 1999; Franchi et al. 2005). The expression of a dominant-negative or a constitutive active form of PKC leads to changes in the WC-1 levels and light responses (Franchi et al. 2005).

Third, the lack of an active WC-1 photocycle means that, once the WCC is activated by light and inhibited by phosphorylation, it cannot be efficiently reused for photoactivation. This is consistent with the results of the two-pulse experiments, which showed that Neurospora is sensitive to a second light pulse given $1 \mathrm{~h}$ after the first one (Schrott 1981; Arpaia et al. 1999; Schwerdtfeger and Linden 2001), a time when the newly synthesized WC-1 protein can be detected (Talora et al. 1999). Thus, the light responses in LL should be mediated mostly by the newly synthesized WC-1 protein. Since the light induction of wc-1 transcription is itself photoadaptive (Figs. 4, 6), the production of the newly synthesized WC-1 protein will be low in LL, which further limits light responses. The limited production of newly synthesized WC-1 and different requirements of WC-1 levels for light induction of different genes should explain the different light-response kinetics for $f r q, v v d$, and al-3 genes. In LL, the light-induced transcription for $f r q$ and $v v d$ is prolonged compared to those of the al genes (Fig. 6; Crosthwaite et al. 1995; Arpaia et al. 1999; Heintzen et al. 2001; Collett et al. 2002; Schwerdtfeger and Linden 2003), probably because the al genes are most sensitive to changes of WC-1 levels for their light induction (Liu et al. 2003).

Finally, factors such as VVD can also play an important role in mediating the photoadaptive responses in Neurospora (Heintzen et al. 2001; Schwerdtfeger and Linden 2001, 2003; Shrode et al. 2001). VVD is a $w c$ dependent LOV-domain containing a blue-light photoreceptor (Cheng et al. 2003a; Schwerdtfeger and Linden 2003). Interestingly, the recombinant VVD also has a very slow photocycle $(\sim 5 \mathrm{~h})$ in vitro. In vvd mutants, light responses are increased and the photoadaptive responses are partially impaired. In addition, the mutants are insensitive to changes in light intensity. Thus, VVD appears to be a general repressor of light responses in Neurospora. How VVD functions is not known. Because VVD and WCs have different cellular localizations, VVD may indirectly affect WC activity after light responses. VVD is expressed at undetectable levels in the dark. Thus, the lack of VVD action in the initial phase of light exposure should also be partly responsible for the strong light responses in the first $15-30 \mathrm{~min}$ in LL. WC-1 hyperphosphorylation in LL is prolonged in $v V d$ mutants (Heintzen et al. 2001; Schwerdtfeger and Linden 2001). In light of the results presented in this study and since WC-1 hyperphosphorylation is also a light response, the prolonged WC-1 hyperphosphorylation in $v v d$ mutants should be the result of enhanced light response of the WC-1 phosphorylation process.

\section{The role of D-WCC phosphorylation in the circadian negative feedback loop}

WC proteins are phosphorylated in the dark. Previously, by mutating the five WC-1 light-independent phosphorylation sites near their Zn-finger regions, we showed that these phosphorylation events are important for its function as the positive element in the circadian negative feedback loop (He et al. 2005). Based on the result, we proposed that the phosphorylation of the WC-1 in the dark negatively regulates its role as a transcriptional activator. This model is further supported by a very recent study suggesting that the FRQ-dependent WC phosphorylation is important for repressing WC activity in DD (Schafmeier et al. 2005). Despite these studies, a direct demonstration of the negative role of the WC dark phosphorylation is lacking. In this study, we provided direct biochemical evidence showing that dephosphorylation of the WCC significantly increased the DNAbinding ability of both D-WCC and L-WCC (Fig. 7A,B). Thus, the WC phosphorylation in the dark is a critical process mediating the closing of the circadian negative feedback loop in Neurospora. Because of the similarities between the circadian negative feedback loops of Neurospora and those of the higher eukaryotic organisms (Young and Kay 2001; Dunlap and Loros 2004), similar mechanisms may be also involved in the regulation of the circadian negative feedback loops in other eukaryotic organisms.

\section{Materials and methods}

Strains and culture conditions

The wild-type strain (87-3 bd, a) was used in this study. A wc$1^{R I P}$ strain (He et al. 2002) was used as the host strain for his-3 targeting a $q a-w c 1-2$ construct (Cheng et al. 2001b). Cultures were grown in minimal medium ( $1 \times$ Vogel's, $2 \%$ glucose $)$ unless specified. Light intensity (1600 lux) was used for all light treatment experiments except for the two pulse experiments, in which 3500 lux was used for the first light pulse. To block protein synthesis, the cultures were first treated with $50 \mu \mathrm{M}$ cycloheximide (CHX) for $1 \mathrm{~h}$ before exposure to light. For induction of WC-1 in the $w c-1^{\mathrm{RIP}}$, qa-WC-1 strain, cultures were grown in liquid medium containing $10 \mathrm{mM}$ QA, $1 \times$ Vogel's, $0.1 \%$ glucose, and $0.17 \%$ arginine for $24 \mathrm{~h}$ in DD. After blotting excess media off mycelia mats, the mats were transferred into $500 \mathrm{~mL}$ of fresh minimal medium ( $2 \%$ glucose) without QA with shaking for $15 \mathrm{~min}$. After blotting off excess media again, the mats were transferred into $500 \mathrm{~mL}$ of fresh minimal medium without QA. Cultures were incubated for $2 \mathrm{~h}$ in DD and 
transferred into $50 \mathrm{~mL}$ of fresh minimal medium before light exposure.

\section{Purification of insect-cell-expressed WC proteins and WCC}

Expression of the individual WC proteins and the WCC in sf9 cells was performed according to the protocol provided by the manufacturer (Bac-to-Bac Baculovirus Expression System; Invitrogen). To obtain the WCC, the insect cells were infected by both a virus expressing WC-1 and a virus expressing His-WC-2. His-tagged proteins were purified with a nickel column. Fluorescence spectra analyses and TLC were performed as previously described (He et al. 2002).

\section{Electrophoretic mobility shift assay}

An electrophoretic mobility shift assay (EMSA) and the purification of nuclear extracts were performed as previously described (Froehlich et al. 2002). The partial purification of WCC from Neurospora tissue ( $w c-2^{K O}$, Myc-His-WC-2) with a nickel column was performed as previously described (He et al. 2002). One microgram of partially purified WCC and $5 \mu \mathrm{g}$ of nuclear extracts were used for EMSA. The probes used for EMSA were vvd probe, ATCCTGCTCCAGATCCCCTACATTACATCTC GATCGACGGCATTC; vvd mutated probe, ATCCTGCTCT CTGTCCCCTACATTACATCTGTCCTGACGGCATTC; al-3 probe, GGCAATATTCGATACCCGCACGCTATGACGATA ATACCGC (Ballario et al. 1996); al-3 mutated probe, GGCA ATATTCACCGCCCGCACGCTATGACGTCCATACCGC; frq probe, CGTCCTGATGCCGCTGCAAGACCGATGACGCTG CAAAATTGAGATCT (Froehlich et al. 2002); and frq mutated probe, CGTCCTTCCTCCGCTGCAAGACCTCTCACGCTG CAAAATTGAGATCT.

\section{Protein and RNA analyses}

Protein analyses were as previously described (Garceau et al. 1997; Cheng et al. 2001a). Equal amounts of total protein (40 $\mu \mathrm{g})$ were loaded in each lane, and after Western blot analyses, the blots were developed by chemiluminescence (ECL; Amersham Pharmacia). RNA extraction and Northern blot analysis were performed as previously described (Crosthwaite et al. 1995). Equal amounts of total RNA $(20 \mu \mathrm{g})$ were loaded in each lane in agarose gels. After electrophoresis, the gels were blotted and probed with RNA probes specific for $v v d, a l-3, f r q, w c-1$, and $r p$-10. Densitometric analyses of the results were performed by using NIH IMAGE 1.61.

\section{ChIP assay}

The ChIP protocol was modified from a published protocol (Johnson et al. 2002). The tissues were fixed in minimal media containing $1 \%$ formaldehyde for $15 \mathrm{~min}$ at room temperature in DD. The immunoprecipitation was performed using our WC-2 antibody (Cheng et al. 2001a). After DNA extraction, the pellets were resuspended in $50 \mu \mathrm{L}$ of TE. Afterward, $2 \mu \mathrm{L}$ of DNA solution was used for PCR. The primer pairs that cover the LREs were as follows: The frq primers are GCCATTCAAGT CAAGCTCGTACCC and GAGCAACGTCCCACATCTTCT AAC. The primers for al-3 are CTCAGCAGGCAAGCA GGGCAAATC and CTAACCCCGTGCGTCGTCTGATCC. The vvd primers are CGCACCCAAGATTACTGTTTCCACC and TAGAGTCGAGGATGAGATGGAG. PCR reactions were $4 \mathrm{~min}$ at $94^{\circ} \mathrm{C}, 26-29$ cycles of $94^{\circ} \mathrm{C}(15 \mathrm{sec}), 60^{\circ} \mathrm{C}(30 \mathrm{sec})$, and $72^{\circ} \mathrm{C}$ for $1 \mathrm{~min}$. PCR products were resolved by electrophoresis on a $2 \%$ agarose gel. Each experiment was independently per- formed at least three times, and immunoprecipitation without the WC-2 antibody was used as the negative control.

\section{Treatment with $\lambda$-phosphatase}

Treatment with $\lambda$-phosphatase was performed in $50 \mu \mathrm{L}$ of reaction containing $10 \mu \mathrm{g}$ of partially purified WCC, $20 \mu \mathrm{g}$ of BSA, and $400 \mathrm{U}$ of $\lambda$-phosphatase (New England Biolabs), with the phosphatase buffer provided by the manufacturer. When used, phosphatase inhibitors $(10 \mathrm{mM}$ sodium orthovanadate and 5 $\mathrm{mM}$ sodium fluoride at $\mathrm{pH} 7.6$ ) were added to the reaction. After treatment for $30 \mathrm{~min}$ at $30^{\circ} \mathrm{C}$, the samples were subjected to an LP. Afterward, $10 \mu \mathrm{L}$ of reaction mixture was used for EMSA. The control samples were treated by the same procedures except for the addition of the $\lambda$-phosphatase.

\section{Acknowledgments}

We thank Lixin Wang for excellent technical assistance, Hongtao $\mathrm{Yu}$ and Bin Li for assistance in insect cell protein expression, and Christian Heintzen for critical reading of the manuscript. This work was supported by grants from the National Institutes of Health (GM068496 and GM062591) and the Welch Foundation to Y.L. Y.L. is the Louise W. Kahn endowed scholar in Biomedical Research at the University of Texas Southwestern Medical Center.

\section{References}

Aronson, B., Johnson, K., Loros, J.J., and Dunlap, J.C. 1994. Negative feedback defining a circadian clock: Autoregulation in the clock gene frequency. Science 263: 1578-1584.

Arpaia, G., Carattoli, A., and Macino, G. 1995. Light and development regulate the expression of the albino-3 gene in Neurospora crassa. Dev. Biol. 170: 626-635.

Arpaia, G., Cerri, F., Baima, S., and Macino, G. 1999. Involvement of protein kinase $\mathrm{C}$ in the response of Neurospora crassa to blue light. Mol. Gen. Genet. 262: 314-322.

Baima, S., Macino, G., and Morelli, G. 1991. Photoregulation of the al-3 gene in Neurospora crassa. Photochem. Photobiol. 11: 107-115.

Ballario, P., Vittorioso, P., Magrelli, A., Talora, C., Cabibbo, A., and Macino, G. 1996. White collar-1, a central regulator of blue-light responses in Neurospora crassa, is a zinc-finger protein. EMBO J. 15: 1650-1657.

Ballario, P., Talora, C., Galli, D., Linden, H., and Macino, G. 1998. Roles in dimerization and blue light photoresponses of PAS and LOV domains of Neurospora crassa white collar proteins. Mol. Micro. 29: 719-729.

Briggs, W.R. and Christie, J.M. 2002. Phototropins 1 and 2: Versatile plant blue-light receptors. Trends Plant Sci. 7: 204210.

Briggs, W.R. and Huala, E. 1999. Blue-light photoreceptors in higher plants. Annu. Rev. Cell Dev. Biol. 15: 33-62.

Carattoli, A., Cogoni, C., Morelli, G., and Macino, G. 1994. Molecular characterization of upstream regulatory sequences controlling the photoinduced expression of the albino-3 gene of Neurospora crassa. Mol. Microbiol. 13: 787795.

Cheng, P., Yang, Y., Heintzen, C., and Liu, Y. 2001a. Coiled-coil domain mediated FRQ-FRQ interaction is essential for its circadian clock function in Neurospora. EMBO J. 20: 101108.

Cheng, P., Yang, Y., and Liu, Y. 2001b. Interlocked feedback loops contribute to the robustness of the Neurospora circa- 
dian clock. Proc. Natl. Acad. Sci. 98: 7408-7413.

Cheng, P., Yang, Y., Gardner, K.H., and Liu, Y. 2002. PAS domain-mediated WC-1/WC-2 interaction is essential for maintaining the steady state level of WC-1 and the function of both proteins in circadian clock and light responses of Neurospora. Mol. Cell. Biol. 22: 517-524.

Cheng, P., He, Q., Yang, Y., Wang, L., and Liu, Y. 2003a. Functional conservation of light, oxygen, or voltage domains in light sensing. Proc. Natl. Acad. Sci. 100: 5938-5943.

Cheng, P., Yang, Y., Wang, L., He, Q., and Liu, Y. 2003b. WHITE COLLAR-1, a multifunctional Neurospora protein involved in the circadian feedback loops, light sensing, and transcription repression of wc-2. J. Biol. Chem. 278: 3801-3808.

Cheng, P., He, Q., He, Q., Wang, L., and Liu, Y. 2005. Regulation of the Neurospora circadian clock by an RNA helicase. Genes \& Dev. 19: 234-241.

Christie, J.M. and Briggs, W.R. 2005. Blue-light sensing and signaling by the phototropins. In Handbook of photosensory receptors (eds. W.R. Briggs and J.L. Spudich), pp. 277-303. Wiley, New York.

Christie, J.M., Salomon, M., Nozue, K., Wada, M., and Briggs, W.R. 1999. LOV (light, oxygen, or voltage) domains of the blue-light photoreceptor phototropin (nph1): Binding sites for the chromophore flavin mononucleotide. Proc. Natl. Acad. Sci. 96: 8779-8783.

Collett, M.A., Garceau, N., Dunlap, J.C., and Loros, J.J. 2002. Light and clock expression of the Neurospora clock gene frequency is differentially driven by but dependent on WHITE COLLAR-2. Genetics 160: 149-158.

Crosson, S. and Moffat, K. 2001. Structure of a flavin-binding plant photoreceptor domain: Insights into light-mediated signal transduction. Proc. Natl. Acad. Sci. 98: 2995-3000.

- 2002. Photoexcited structure of a plant photoreceptor domain reveals a light-driven molecular switch. Plant Cell 14: 1067-1075.

Crosson, S., Rajagopal, S., and Moffat, K. 2003. The LOV domain family: Photoresponsive signaling modules coupled to diverse output domains. Biochemistry 42: 2-10.

Crosthwaite, S.K., Loros, J.J., and Dunlap, J.C. 1995. Light-induced resetting of a circadian clock is mediated by a rapid increase in frequency transcript. Cell 81: 1003-1012.

Crosthwaite, S.K., Dunlap, J.C., and Loros, J.J. 1997. Neurospora $w c-1$ and $w c-2$ : Transcription, photoresponses, and the origins of circadian rhythmicity. Science 276: 763-769.

Degli Innocenti, F. and Russo, V.E.A. 1984. Genetic analysis of blue light-induced responses in Neurospora crassa. In Blue light effects in biological systems (ed. H. Senger), pp. 213219. Springer Verlag, Berlin.

Denault, D.L., Loros, J.J., and Dunlap, J.C. 2001. WC-2 mediates WC-1-FRQ interaction within the PAS protein-linked circadian feedback loop of Neurospora. EMBO J. 20: 109-117.

Dunlap, J.C. and Loros, J.J. 2004. The Neurospora circadian system. J. Biol. Rhythms 19: 414-424.

Franchi, L., Fulci, V., and Macino, G. 2005. Protein kinase C modulates light responses in Neurospora by regulating the blue light photoreceptor WC-1. Mol. Microbiol. 56: 334-345.

Froehlich, A.C., Liu, Y., Loros, J.J., and Dunlap, J.C. 2002. White Collar-1, a circadian blue light photoreceptor, binding to the frequency promoter. Science 297: 815-819.

Froehlich, A.C., Loros, J.J., and Dunlap, J.C. 2003. Rhythmic binding of a WHITE COLLAR-containing complex to the frequency promoter is inhibited by FREQUENCY. Proc. Natl. Acad. Sci. 100: 5914-5919.

Garceau, N., Liu, Y., Loros, J.J., and Dunlap, J.C. 1997. Alternative initiation of translation and time-specific phosphorylation yield multiple forms of the essential clock protein FRE-
QUENCY. Cell 89: 469-476.

Gorl, M., Merrow, M., Huttner, B., Johnson, J., Roenneberg, T., and Brunner, M. 2001. A PEST-like element in FREQUENCY determines the length of the circadian period in Neurospora crassa. EMBO J. 20: 7074-7084.

Harper, S.M., Neil, L.C., and Gardner, K.H. 2003. Structural basis of a phototropin light switch. Science 301: 1541-1544.

He, Q., Cheng, P., Yang, Y., Wang, L., Gardner, K.H., and Liu, Y. 2002. White collar-1, a DNA binding transcription factor and a light sensor. Science 297: 840-843 (published as ScienceExpress online in advance).

He, Q., Cheng, P., Yang, Y., He, Q., Yu, H., and Liu, Y. 2003. FWD1-mediated degradation of FREQUENCY in Neurospora establishes a conserved mechanism for circadian clock regulation. EMBO J. 22: 4421-4430.

He, Q., Shu, H., Cheng, P., Chen, S., Wang, L., and Liu, Y. 2005. Light-independent phosphorylation of WHITE COLLAR-1 regulates its function in the Neurospora circadian negative feedback loop. J. Biol. Chem. 280: 17526-17532.

Heintzen, C., Loros, L.L., and Dunlap, J.C. 2001. The PAS protein VIVID defines a clock-associated feedback loop that represses light input, modulates gating and regulates clock resetting. Cell 104: 453-464.

Imaizumi, T., Tran, H.G., Swartz, T.E., Briggs, W.R., and Kay, S.A. 2003. FKF1 is essential for photoperiodic-specific light signalling in Arabidopsis. Nature 426: 302-306.

Johnson, L., Cao, X., and Jacobsen, S. 2002. Interplay between two epigenetic marks. DNA methylation and histone $\mathrm{H} 3$ lysine 9 methylation. Curr. Biol. 12: 1360-1367.

Lakin-Thomas, P., Coté, G., and Brody, S. 1990. Circadian rhythms in Neurospora: Biochemistry and genetics. Crit. Rev. Micro. 17: 365-416.

Lee, K., Loros, J.J., and Dunlap, J.C. 2000. Interconnected feedback loops in the Neurospora circadian system. Science 289: $107-110$.

Lee, K., Dunlap, J.C., and Loros, J.J. 2003. Roles for WHITE COLLAR-1 in circadian and general photoperception in Neurospora crassa. Genetics 163: 103-114.

Linden, H. and Macino, G. 1997. White collar-2, a partner in blue-light signal transduction, controlling expression of light-regulated genes in Neurospora crassa. EMBO J. 16: 98 109.

Linden, H., Ballario, P., and Macino, G. 1997. Blue light regulation in Neurospora crassa. Fungal Genet. Biol. 22: 141-150.

Liu, Y. 2003. Molecular mechanisms of entrainment in the Neurospora circadian clock. J. Biol. Rhythms 18: 195-205.

Liu, Y., Loros, J., and Dunlap, J.C. 2000. Phosphorylation of the Neurospora clock protein FREQUENCY determines its degradation rate and strongly influences the period length of the circadian clock. Proc. Natl. Acad. Sci. 97: 234-239.

Liu, Y., He, Q., and Cheng, P. 2003. Photoreception in Neurospora: A tale of two White Collar proteins. Cell Mol. Life Sci. 60: $2131-2138$.

Mas, P., Kim, W.Y., Somers, D.E., and Kay, S.A. 2003. Targeted degradation of TOC1 by ZTL modulates circadian function in Arabidopsis thaliana. Nature 426: 567-570.

Pugh Jr., E.N., Nikonov, S., and Lamb, T.D. 1999. Molecular mechanisms of vertebrate photoreceptor light adaptation. Curr. Opin. Neurobiol. 9: 410-418.

Salomon, M., Christie, J.M., Knieb, E., Lempert, U., and Briggs, W.R. 2000. Photochemical and mutational analysis of the FMN-binding domains of the plant blue light receptor, phototropin. Biochemistry 39: 9401-9410.

Schafmeier, T., Haase, A., Kaldi, K., Scholz, J., Fuchs, M., and Brunner, M. 2005. Transcriptional feedback of neurospora circadian clock gene by phosphorylation-dependent inacti- 
vation of its transcription factor. Cell 122: 235-246.

Schrott, E. 1981. The biphasic fluence response of carotenogenesis in Neurospora crassa: Temporal insensitivity of the photoreceptor system. Planta 151: 371-374.

Schwerdtfeger, C. and Linden, H. 2000. Localization and lightdependent phosphorylation of white collar 1 and 2, the two central components of blue light signaling in Neurospora crassa. Eur. J. Biochem. 267: 414-422.

. 2001. Blue light adaptation and desensitization of light signal transduction in Neurospora crassa. Mol. Microbiol. 39: $1080-1087$.

. 2003. VIVID is a flavoprotein and serves as a fungal blue light photoreceptor for photoadaptation. EMBO J. 22: 48464855.

Shrode, L.B., Lewis, Z.A., White, L.D., Bell-Pedersen, D., and Ebbole, D.J. 2001. vvd is required for light adaptation of conidiation-specific genes of Neurospora crassa, but not circadian conidiation. Fungal Genet. Biol. 32: 169-181.

Somers, D.E., Schultz, T.F., Milnamow, M., and Kay, S.A. 2000. ZEITLUPE encodes a novel clock-associated PAS protein from Arabidopsis. Cell 101: 319-328.

Talora, C., Franchi, L., Linden, H., Ballario, P., and Macino, G. 1999. Role of a white collar-1-white collar-2 complex in blue-light signal transduction. EMBO J. 18: 4961-4968.

Yang, Y., Cheng, P., and Liu, Y. 2002. Regulation of the Neurospora circadian clock by casein kinase II. Genes \& Dev. 16: 994-1006.

Yang, Y., He, Q., Cheng, P., Wrage, P., Yarden, O., and Liu, Y. 2004. Distinct roles for PP1 and PP2A in the Neurospora circadian clock. Genes \& Dev. 18: 255-260.

Young, M.W. and Kay, S.A. 2001. Time zones: A comparative genetics of circadian clocks. Nat. Rev. Genet. 2: 702-715. 


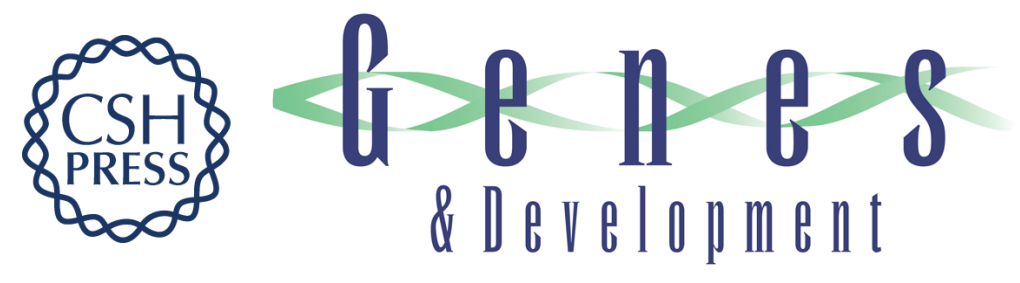

\section{Molecular mechanism of light responses in Neurospora: from light-induced transcription to photoadaptation}

Qiyang He and Yi Liu

Genes Dev. 2005, 19:

Access the most recent version at doi:10.1101/gad.1369605

References This article cites 57 articles, 28 of which can be accessed free at: http://genesdev.cshlp.org/content/19/23/2888.full.html\#ref-list-1

License

Email Alerting

Receive free email alerts when new articles cite this article - sign up in the box at the top Service right corner of the article or click here.

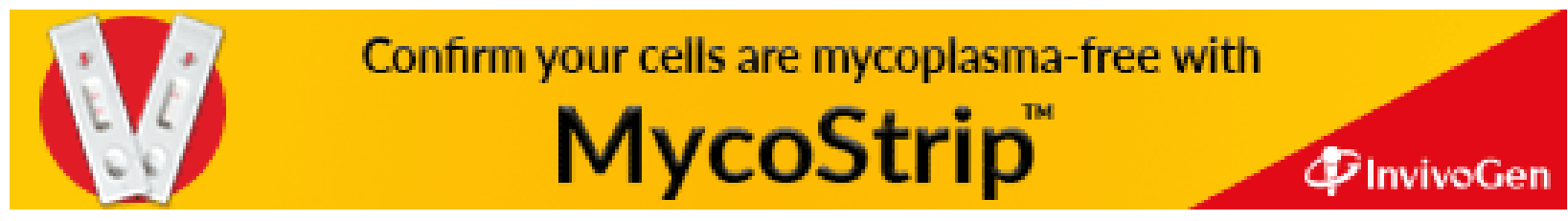

